\title{
Characteristic and Outcome of Psoriatic Arthritis Patients with Hyperuricemia
}

\author{
Roa'A AlJohani, Ari Polachek, Justine Yang Ye, Vinod Chandran, and Dafna D. Gladman
}

\begin{abstract}
Objective. To determine the characteristics of patients with psoriatic arthritis (PsA) who have hyperuricemia (HUC) and their outcomes, especially cardiovascular (CVD) and kidney diseases.

Methods. Patients have been followed prospectively at the PsA clinic according to a standard protocol at 6- to 12-month intervals. We defined HUC in men $>450 \mu \mathrm{mol} / 1$ or women $>360 \mu \mathrm{mol} / 1$. We matched patients with HUC based on sex and age \pm 5 years with normal uric acid patients. Demographics information and disease characteristics were reviewed. Outcomes of patients with HUC, especially CVD and kidney diseases, were recorded. Conditional logistic regression was performed to determine factors independently associated with HUC in patients with PsA.

Results. There were 325 (31.9\%) out of 1019 patients with PsA who had HUC. Of these, 318 cases were matched to 318 controls. There were 11 (3.4\%) out of 325 patients with HUC who had gout. Patients with HUC had longer disease duration and a higher Psoriasis Area and Severity Index. They had more concurrent comorbidities, including CVD and metabolic diseases, as well as higher prevalence of kidney stones and higher creatinine. Only 1 patient with HUC was treated with allopurinol at first evaluation visit and 7 patients during followup. Over the followup, 163 of the 318 patients had persistent HUC (pHUC) for more than 2 visits. Patients with pHUC developed more myocardial infarction, heart failure, and renal impairment. Multivariate analysis showed an association between pHUC, PsA disease duration, and obesity.

Conclusion. HUC is common in patients with PsA, especially in those with longer disease duration and obesity. Proper control of HUC and metabolic diseases may play a preventive role in improving PsA outcomes. (First Release December 1 2017; J Rheumatol 2018;45:213-17; doi:10.3899/ jrheum.170384)
\end{abstract}

Key Indexing Terms:

PSORIATIC ARTHRITIS HYPERURICEMIA CARDIOVASCULAR DISEASE OUTCOMES

Psoriatic arthritis (PsA) is an inflammatory arthritis associated with psoriasis and affects $20-30 \%$ of patients with psoriasis $^{1,2}$. Although PsA was initially considered a benign

From the University of Toronto Lupus Clinic, Toronto Western Hospital, Centre for Prognosis Studies in the Rheumatic Diseases, University Health Network, Toronto, Ontario, Canada; Department of Medicine, Taibah University, Medina, Saudi Arabia; Department of Medicine, Division of Rheumatology, University of Toronto, Toronto, Ontario, Canada.

R. AlJohani, MD, Clinical and Research Fellow, University of Toronto Lupus Clinic, Toronto Western Hospital, Centre for Prognosis Studies in the Rheumatic Diseases, and Department of Medicine, Taibah University; A. Polachek, MD, Clinical and Research Fellow, University of Toronto Lupus Clinic, Toronto Western Hospital, Centre for Prognosis Studies in the Rheumatic Diseases; J.Y. Ye, MSc, Biostatistician, Centre for Prognosis Studies in the Rheumatic Diseases, Toronto Western Hospital; V. Chandran, MBBS, MD, DM, PhD, Assistant Professor, University of Toronto, Department of Medicine, Division of Rheumatology, University of Toronto, and Co-Director, Psoriatic Arthritis Program, Centre for

Prognosis Studies in the Rheumatic Diseases, Toronto Western Hospital, University Health Network; D.D. Gladman, MD, FRCPC, Director,

Psoriatic Arthritis Program, Centre for Prognosis Studies in the Rheumatic Diseases, and Senior Scientist, Krembil Research Institute, Toronto Western Hospital, University Health Network.

Address correspondence to Dr. D.D. Gladman, University of Toronto

Psoriatic Arthritis Program, Centre for Prognosis Studies in the

Rheumatic Diseases, Krembil Research Institute, University Health

Network, 399 Bathurst St. 1E-410B, Toronto, Ontario M5T 2S8, Canada.

E-mail:dafna.gladman@utoronto.ca

Accepted for publication September 12, 2017. disease, it is now recognized that PsA can lead to severe joint damage and deformities and significantly affect function, quality of life, and work productivity ${ }^{3,4}$. Further, most of the patients with PsA have associated comorbidities (which significantly lower quality of life $\mathrm{e}^{5,6}$ ), as well as increased risk of morbidity and mortality ${ }^{7}$.

A comorbidity associated with PsA is hyperuricemia (HUC). The prevalence of asymptomatic HUC in the general population is estimated at $2-13 \%$, while in psoriatic patients, it is 3 times greater ${ }^{8}$. The higher prevalence in psoriatic patients may be related to increased cell turnover, as well as the known systemic inflammation associated with the disease $^{9}$.

Although several studies have shown a correlation between HUC and psoriasis, results were conflicting ${ }^{10,11,12}$. It is not clear whether HUC is correlated with the extent of skin involvement, or whether it results from the effects of coexisting features of the metabolic syndrome. In 2000, Bruce, et al ${ }^{13}$ found HUC in $20.7 \%$ of patients with PsA, and it reflected mainly metabolic changes. On the other hand, a Korean study showed a correlation between the severity of skin involvement and high uric acid level ${ }^{14}$.

HUC not only causes gout but also stimulates systemic inflammation that contributes to the development and patho-

Personal non-commercial use only. The Journal of Rheumatology Copyright @ 2018 . All rights reserved. 
genesis of cardiovascular (CVD) and renal diseases ${ }^{15}$. Moreover, previous studies confirmed that elevated uric acid level is predictive of severe subclinical atherosclerosis in patients with PsA ${ }^{16,17}$.

The aim of our study was to determine the prevalence and characteristics of psoriatic patients with HUC and to determine the adverse effect of HUC on outcomes, particularly CVD and renal diseases.

\section{MATERIALS AND METHODS}

Setting. The University of Toronto Psoriatic Arthritis Clinic was established in 1978. The clinic serves as a referral center and follows patients in a prospective manner according to standard protocol ${ }^{15}$. Patients are included in the cohort if they have psoriasis and inflammatory arthritis; other types of arthritis have been excluded ${ }^{18}$. There are $98 \%$ of patients in the clinic who fulfill the ClASsification of Psoriatic ARthritis criteria (CASPAR) for the classification of PsA ${ }^{19}$. Each patient is assessed at 6- to 12-month intervals. At each visit, a complete history, physical examination, and laboratory assessment are performed. All data are stored in a Web-based database. Written consent from all subjects was obtained according to the Declaration of Helsinki. The study was approved by the research ethics board of the University Health Network (REB 08-0630-AE).

Patient selection. Only patients followed from 2006 were included because data on body mass index (BMI) were not recorded before 2006. We defined HUC as male $>450 \mu \mathrm{mol} / 1$ or women $>360 \mu \mathrm{mol} / 1$. Patients with PsA and HUC at any clinic visit were selected as cases, while patients with PsA who have had normal uric acid level at all assessments and were matched for age $( \pm 5$ yrs) and sex served as controls.

Measures. Demographic data were collected, including age, sex, race, ethnicity, duration of PsA and psoriasis, employment and education level, smoking status, alcohol consumption, and postmenopausal status. Comorbidities were also recorded, including hypertension (HTN; systolic $>140$ and/or diastolic > 90), diabetes mellitus, hypercholesterolemia (> 5.2 $\mathrm{mmol} / \mathrm{l})$, hypertriglyceridemia $(>3.2 \mathrm{mmol} / \mathrm{l})$, ischemic heart disease, kidney stones, and cancer. Clinical assessments including BMI, psoriatic area severity index (PASI), tender and swollen joint counts, and spine metrology were collected. Laboratory results included levels of uric acid, C-reactive protein (CRP), erythrocyte sedimentation rate (ESR), creatinine, aspartate transaminase (AST), and alanine transaminase (ALT). Medication history, including usage of urate-lowering therapy, was recorded.

Outcomes. We identified patients with persistent HUC (pHUC, high uric acid level for more than 2 visits) and determined new development of CVD and kidney disease. CVD were defined as the following: (1) myocardial infarction (MI), characterized by definite electrocardiographic abnormalities, typical symptoms with probable electrocardiographic abnormalities and abnormal enzymes, or typical symptoms and abnormal enzymes; (2) angina pectoris, characterized by chest discomfort that was aggravated by physical activity, relieved by rest or by the use of nitroglycerine, and confirmed by a cardiologist or abnormal findings on myocardial perfusion study or angiography; (3) congestive heart failure, identified from typical symptoms and signs on physical examination, abnormal chest radiograph, or abnormal finding on echocardiogram; and (4) cardiomyopathy, including restrictive, dilated, or hypertrophic heart muscles. Kidney disease was defined as raised serum creatinine $>120 \mu \mathrm{mol} / \mathrm{l}$ or the presence of kidney stones as determined by patient report.

Statistical analysis. The demographic variables and baseline characteristics were reported with descriptive statistics to describe the prevalence of HUC. In the descriptive analysis, continuous variables were compared using 1-way ANOVA; categorical variables were compared using chi-square test. All continuous variables were plotted into a quantile-quantile plot for normality check. All non-normal variables were reported as median and used Kruskal-Wallis rank sum test for comparison.
Control patients with normal uric acid level (NUC) were selected from the same PsA cohort and matched 1:1 with case group (HUC) by sex and baseline age $( \pm 5 \mathrm{yrs})$. A multivariate conditional logistic regression was used to analyze the difference between HUC versus NUC, with the following covariates in the full model: PsA duration, psoriasis duration, smoking status, elevated ESR (> $20 \mathrm{~mm} / \mathrm{h}$ for women, > $15 \mathrm{~mm} / \mathrm{h}$ for men), HTN, kidney stone, diabetes, PASI score, elevated creatinine, elevated AST, elevated ALT, and BMI. A backward stepwise elimination method was then performed to find significant predictors for a reduced model.

Further, patients with $>2$ consecutive visits with high uric acid level were selected from the HUC cohort (pHUC). A descriptive summary was performed between the pHUC group versus the NUC group for the following variables: elevated creatinine, angina, cardiomyopathy, MI, congestive heart failure, and kidney stones. To keep consistency with previous multivariable regression analysis, the same matched control patients were selected for full and reduced models.

The threshold for a statistically significance difference was set at $p$ $<0.05$. SAS (version 9.3, SAS Institute) was used for all statistical analyses.

\section{RESULTS}

Among 1019 patients with PsA who had a followup in our clinic from 2006, 318 patients (31.2\%) with high serum uric acid (HUC) and 318 control patients with normal uric acid (NUC) were included. They were matched 1:1 [based on sex and age $( \pm 5 \mathrm{yrs})]$. Their baseline characteristics are shown in Table 1. There was no significant difference in baseline characteristics including age, sex, ethnicity, education, and employment between patients with HUC and NUC. There were $11(3.5 \%)$ out of 318 patients with HUC who had gout. Smoking was higher in patients with HUC $(\mathrm{p}=0.021)$, but alcohol consumption was similar between the 2 groups. Patients with HUC appeared to have longer disease duration of PsA ( $\mathrm{p}<0.001)$ and psoriasis $(\mathrm{p}=0.0016)$ compared to patients with NUC. PASI was significantly higher in patients with HUC $(p=0.006)$. No statistical difference in swollen or tender joint counts was found between the 2 groups. Inflammatory markers including ESR and CRP were higher in the HUC group. Compared to patients with NUC, patients with HUC had more associated comorbidities, more CVD including HTN $(\mathrm{p}<0.001)$ and angina $(\mathrm{p}=0.037)$. Also, the prevalence of diabetes mellitus $(\mathrm{p}=0.0042)$, obesity ( $\mathrm{p}$ $<0.001)$, and elevated liver function tests $(\mathrm{p}=0.01)$ were higher in the HUC group. There was no statistically significant difference in hypercholesterolemia and hypertriglyceridemia. Kidney diseases including high serum creatinine, and kidney stones were also higher in the HUC group compared to patients with NUC. No significant difference in cancer between the 2 groups was identified (data not shown). Also, there was no statistically significant difference between the 2 groups in the treatment modalities including use of nonsteroidal antiinflammatory drugs, disease-modifying antirheumatic drugs, and biologics.

Over the followup, 163 of the 318 patients had pHUC on more than 2 consecutive visits. Patients with pHUC developed more MI $(p=0.008)$ and congestive heart failure $(\mathrm{p}<0.001)$. No statistically significant difference was found in angina and cardiomyopathy between the 2 groups. Also,

Personal non-commercial use only. The Journal of Rheumatology Copyright $\odot$ (2018. All rights reserved 
Table 1. Characteristics of the study population at clinic entry. Values are $\mathrm{n}$ $(\%)$ or mean $\pm \mathrm{SD}$, unless otherwise specified.

\begin{tabular}{|c|c|c|c|}
\hline Covariate & NUC, $n=318$ & HUC, $n=318$ & $\mathrm{p}$ \\
\hline Sex & & & 1 \\
\hline Female & $126(40)$ & $126(40)$ & \\
\hline Male & $192(60)$ & $192(60)$ & \\
\hline Age & $51.4 \pm 13.3$ & $53.4 \pm 13.7$ & 0.059 \\
\hline Median (min, max) & $52(18.2,88.2)$ & $54.2(17.5,85.2)$ & \\
\hline Race & & & 0.67 \\
\hline White & $268(84)$ & $262(82)$ & \\
\hline Others & $50(16)$ & $54(17)$ & \\
\hline PsA duration & $8.4 \pm 8.6$ & $13.9 \pm 11.9$ & $<0.001$ \\
\hline Psoriasis duration & $20 \pm 14.5$ & $23.7 \pm 14.1$ & 0.0016 \\
\hline Duration of followup & $3.6 \pm 2.9$ & $6.1 \pm 3.1$ & \\
\hline Median & 3.02 & 6.5 & \\
\hline Employment & & & 0.57 \\
\hline Employed & $188(59)$ & $196(62)$ & \\
\hline None & $130(41)$ & $122(38)$ & \\
\hline Smoking & $132(42)$ & $162(51)$ & 0.021 \\
\hline Alcohol intake & & & 0.61 \\
\hline Daily & $27(9)$ & $32(10)$ & \\
\hline Social & $152(48)$ & $140(44)$ & \\
\hline Hypertension & $77(24)$ & $141(44)$ & $<0.001$ \\
\hline Angina & $2(1)$ & $10(3)$ & 0.037 \\
\hline Cardiomyopathy & $1(0)$ & $1(0)$ & 1 \\
\hline Myocardial infarction & $1(0)$ & $4(1)$ & 0.37 \\
\hline Congestive heart failure & $0(0)$ & $4(1)$ & 0.12 \\
\hline High creatinine & $6(2)$ & $26(8)$ & $<0.001$ \\
\hline Kidney stones & $9(3)$ & $28(9)$ & 0.0019 \\
\hline Diabetes mellitus & $25(8)$ & $48(15)$ & 0.0042 \\
\hline Swollen joints count & $0.4 \pm 1.4$ & $0.6 \pm 1.9$ & 0.21 \\
\hline Tender joints count & $2.8 \pm 6.8$ & $3 \pm 6.3$ & 0.76 \\
\hline PASI & $3.3 \pm 5.4$ & $4.7 \pm 7.3$ & 0.0062 \\
\hline $\begin{array}{l}\mathrm{ESR},>20 \mathrm{~mm} / \mathrm{h} \text { for men, } \\
>>15 \mathrm{~mm} / \mathrm{h} \text { for women }\end{array}$ & $76(24)$ & $130(41)$ & $<0.001$ \\
\hline $\mathrm{CRP}, \mathrm{mg} / \mathrm{l}$ & $57 \pm 29$ & $89 \pm 49$ & $<0.001$ \\
\hline Hypercholesterolemia & $54(17)$ & $60(19)$ & 1 \\
\hline Hypertriglyceridemia & $14(4)$ & $13(4)$ & 0.7 \\
\hline BMI & $28.5 \pm 6.5$ & $31.7 \pm 5.7$ & $<0.001$ \\
\hline
\end{tabular}

BMI: body mass index; CRP: C-reactive protein; ESR: erythrocyte sedimentation rate; HUC: hyperuricemia; NUC: normal uric acid; PASI: Psoriasis Area and Severity Index; PsA: psoriatic arthritis.

kidney diseases, including high serum creatinine, developed more often in patients with pHUC (Table 2). Regarding use of urate-lowering therapy, only 1 patient with HUC was taking allopurinol at first visit, and 7 patients were treated with allopurinol over the followup.

Multivariable analysis. Multivariable analysis revealed these variables to be independent predictors for HUC in patients with PsA: kidney stones (OR 4.430, 95\% CI 0.50-29.24) and BMI (OR 1.085, 95\% CI 1.027-1.146, Table 3). On multivariable analysis for patients with $\mathrm{pHUC}$, PsA disease duration (OR 1.073, 95\% CI 1.028-1.113) and BMI (OR $1.176,95 \%$ CI $1.062-1.303)$ were predictors for pHUC (Table 4).
Table 2. Outcomes of PsA patients with persistent HUC. Values are n (\%).

\begin{tabular}{lccc}
\hline Variables & $\begin{array}{c}\text { NUC, } \\
\mathrm{n}=318\end{array}$ & $\begin{array}{c}\text { Persistent HUC, } \\
\mathrm{n}=163\end{array}$ & $\mathrm{p}$ \\
\hline Serum creatinine, $>120 \mu \mathrm{mol} / \mathrm{l}$ & $14(4)$ & $30(18)$ & $\mathbf{< 0 . 0 0 1}$ \\
Angina & $4(1)$ & $7(4)$ & 0.07 \\
Cardiomyopathy & $1(0)$ & $3(2)$ & 0.12 \\
Myocardial infarction & $1(0)$ & $3(2)$ & $\mathbf{0 . 0 0 8}$ \\
Congestive heart failure & $0(0)$ & $8(5)$ & $\mathbf{0 . 0 0 1}$ \\
Kidney stones & $23(7)$ & $20(12)$ & 0.19
\end{tabular}

Data in bold face are statistically significant. HUC: hyperuricemia; NUC: normal uric acid; PsA: psoriatic arthritis.

\section{DISCUSSION}

Literature highlights the significant association between high serum uric acid level and psoriasis. In our study, we initially investigated the prevalence and characteristics of PsA patients with HUC. We found the prevalence of HUC in patients with PsA is $32 \%$, higher than previously reported $^{8,20,21}$.

Additionally, we showed that patients with HUC had a higher PASI score and more associated metabolic diseases. An earlier report from our cohort had shown that the extent of skin involvement was not associated with HUC but was mainly associated with metabolic changes ${ }^{13}$. The extent of skin involvement, as measured by the PASI score, was not associated with HUC in the current study. Previous literature has provided some conflicting results as to whether HUC is related to the severity of psoriasis ${ }^{8,10,11}$ or metabolic dysregulation $^{21,22}$. It was reported that the serum uric acid is 1 of the determinants of the metabolic syndrome in nonpsoriatic patients; with 1 unit increase of serum uric acid, the odds of developing metabolic syndrome roughly doubled ${ }^{22}$. Most of the literature is in favor of metabolic dysregulation as a reflecting factor for HUC.

Importantly, we found that PsA disease duration, obesity, and kidney stones were independent predictors for HUC in patients with PsA. Prolonged disease duration in PsA patients with chronic inflammatory diseases may promote the release of proinflammatory cytokines and tumor necrosis factor, which are positively associated with high uric acid level ${ }^{23,24}$. A recent large Korean cohort study showed that an increased serum uric acid was modestly and independently associated with increased risk of development of nephrolithiasis, after adjustment for hemostasis model assessment of insulin resistance and high-sensitivity $\mathrm{CRP}^{25}$. Additionally, a previous study confirmed the association between obesity ( 1 of the metabolic syndrome components) and HUC in psoriatic patients, which is in agreement with our results ${ }^{8}$. In our study, we did not identify psoriasis severity as a predictor for developing HUC. A study by Scarpa, et $a^{26}$ identified PsA patients without psoriasis but unfortunately did not document whether the patients had HUC.

Treatment of HUC is a potential target for preventing the Personal non-commercial use only. The Journal of Rheumatology Copyright $\odot$ 2018. All rights reserved. 
Table 3. Conditional logistic regression for matched pairs of 318 patients with at least 1 visit of HUC: a multivariable analysis.

\begin{tabular}{lcccc}
\hline Effect & OR $(95 \% \mathrm{CI})$ & $\mathrm{p}$ & Multivariate Reduced, OR $(95 \% \mathrm{CI})$ & $\mathrm{p}$ \\
\hline PsA duration & $1.059(1.022-1.098)$ & 0.001 & $1.066(1.03-1.1)$ & $<0.0001$ \\
Psoriasis duration & $1.017(0.991-1.043)$ & 0.198 & & \\
Smoking & $1.078(0.563-2.062)$ & 0.821 & & \\
High ESR & $1.492(0.728-3.057)$ & 0.274 & & \\
HTN & $1.071(0.495-2.314)$ & 0.862 & & \\
Kidney stones & $4.430(0.50-39.24)$ & 0.181 & & \\
Diabetes & $1.321(0.368-4.740)$ & 0.669 & & \\
PASI & $1.007(0.952-1.065)$ & 0.815 & & \\
High creatinine & $2.328(0.196-27.724)$ & 0.503 & & \\
High AST & $1.423(0.553-3.664)$ & 0.464 & $2.59(1.06-6.32)$ & 0.036 \\
High ALT & $1.754(0.588-5.232)$ & 0.313 & $1.095(1.04-1.15)$ & 0.005 \\
BMI & $1.085(1.027-1.146)$ & 0.003 & \\
\hline
\end{tabular}

AST: aspartate aminotransferase; ALT: alanine aminotransferase; BMI: body mass index; ESR: erythrocyte sedimentation rate; HTN: hypertension; HUC: hyperuricemia; PASI: Psoriasis Area and Severity Index; PsA: psoriatic arthritis.

Table 4. Conditional logistic regression for matched pairs of 163 patients with persistent HUC: a multivariable analysis.

\begin{tabular}{lcccc}
\hline Effect & OR $(95 \% \mathrm{CI})$ & $\mathrm{p}$ & Multivariate Reduced, OR (95\% CI) & $\mathrm{p}$ \\
\hline PsA duration & $1.073(1.02-1.13)$ & 0.007 & $1.06(1.017-1.107)$ & 0.006 \\
Psoriasis duration & $0.99(0.957-1.042)$ & 0.947 & & \\
Smoking & $0.728(0.247-2.149)$ & 0.565 & & \\
High ESR & $0.806(0.208-3.123)$ & 0.754 & & \\
PASI & $1.082(0.974-1.203)$ & 0.14 & & \\
High creatinine & $5.349(0.164-174.139)$ & 0.345 & & \\
High AST & $0.467(0.091-2.396)$ & 0.345 & & \\
High ALT & $3.943(0.630-24.6)$ & 0.142 & $1.160(1.066-1.263)$ & \\
BMI & $1.176(1.062-1.303)$ & 0.0018 & & \\
\hline
\end{tabular}

AST: aspartate aminotransferase; ALT: alanine aminotransferase; BMI: body mass index; ESR: erythrocyte sedimentation rate; HUC: hyperuricemia; PASI: Psoriasis Area and Severity Index; PsA: psoriatic arthritis.

incidence of CVD and kidney diseases. Previous studies have shown a correlation between CVD and kidney diseases in PsA patients with $\mathrm{HUC}^{13,27,28}$. However, it remains to be determined whether the relationship between HUC, cardiovascular, and kidney events is related only to the elevated level of uric acid or whether it is a combination of metabolic syndrome and HUC.

Several studies showed that the risk of developing cardiovascular events in patients with PsA is higher than in the general population and may be higher than in people with psoriasis alone ${ }^{29,30,31}$. Importantly, Gonzalez-Gay, et al showed in their study that in patients with PsA, there was a significant association between high uric acid level and subclinical atherosclerosis in patients without CVD risk factors ${ }^{17}$. We found in our study increased prevalence of CVD in PsA patients with pHUC, especially MI and congestive heart failure.

Moreover, a previous metaanalysis has found an elevated serum uric acid was positively associated with new-onset kidney disease in populations with normal baseline renal function ${ }^{31}$. Similarly, we found an increased serum creatinine in PsA patients with pHUC.

Whether we should treat asymptomatic HUC in patients with PsA is not clear. An interesting study by Wei, et $a l^{32}$, which included a large number of nonpsoriatic patients taking allopurinol for HUC, found that higher doses of allopurinol were associated with better control of urate and lower risks of both cardiovascular events and mortality in all patients taking allopurinol treatment. In our study, only a small number of patients were treated with allopurinol $(2.5 \%)$; thus, we cannot comment on whether treatment with urate-lowering therapy improves CVD and kidney diseases. However, we showed in our study higher prevalence of CVD and kidney impairments in patients with pHUC. Thus, controlling metabolic diseases and HUC in psoriatic patients may play a preventive role in improving PsA outcomes.

Personal non-commercial use only. The Journal of Rheumatology Copyright $\subset$ 2018. All rights reserved. 
Our study demonstrates that almost one-third of the patients with PsA have HUC and half of those have pHUC, but only a few develop clinical gout. These patients are at risk for developing metabolic syndrome. It is therefore important to screen patients with PsA for HUC and it may be necessary to treat it to improve the outcomes in these patients.

\section{REFERENCES}

1. Ibrahim G, Waxman R, Helliwell PS. The prevalence of psoriatic arthritis in people with psoriasis. Arthritis Rheum 2009;61:1373-8.

2. Gladman DD, Antoni C, Mease P, Clegg DO, Nash P. Psoriatic arthritis: epidemiology, clinical features, course, and outcome. Ann Rheum Dis 2005;64 Suppl 2:ii14-7.

3. Husted JA, Tom BD, Farewell VT, Schentag CT, Gladman DD. Description and prediction of physical functional disability in psoriatic arthritis: a longitudinal analysis using a Markov model approach. Arthritis Rheum 2005;53:404-9.

4. Tillett W, de-Vries C, McHugh NJ. Work disability in psoriatic arthritis: a systematic review. Rheumatology 2012;51:275-83.

5. Husted JA, Thavaneswaran A, Chandran V, Gladman DD. Incremental effects of comorbidity on quality of life in patients with psoriatic arthritis. J Rheumatol 2013;40:1349-56.

6. Sanchez-Carazo JL, Lopez-Estebaranz JL, Guisado C. Comorbidities and health-related quality of life in Spanish patients with moderate to severe psoriasis: a cross-sectional study (Arizona study). J Dermatol 2014;41:673-8.

7. Gladman DD. Mortality in psoriatic arthritis. Clin Exp Rheumatol 2008;5 Suppl 51:S62-5.

8. Gisondi P, Targher G, Cagalli A, Girolomoni G. Hyperuricemia in patients with chronic plaque psoriasis. J Am Acad Dermatol 2014;70:127-30

9. Merola JF, Wu S, Han J, Choi HK, Qureshi AA. Psoriasis, psoriatic arthritis and risk of gout in US men and women. Ann Rheum Dis 2015;74:1495-500.

10. Yilmaz E, Tamer E, Artuz F, Külcü Çakmak S, Kokturk F. Evaluation of serum uric acid levels in psoriasis vulgaris. Turk $\mathrm{J}$ Med Sci 2017;47:531-4.

11. Li X, Miao X, Wang H, Wang Y, Li F, Yang Q, et al. Association of serum uric acid levels in psoriasis: a systematic review and meta-analysis. Medicine 2016;95:e3676.

12. Lai YC, Yew YW. Psoriasis and uric acid: a population-based cross-sectional study. Clin Exp Dermatol 2016;41:260-6.

13. Bruce IN, Schentag CT, Gladman DD. Hyperuricemia in psoriatic arthritis: prevalence and associated features. J Clin Rheumatol 2000;6:6-9.

14. Kwon HH, Kwon IH, Choi JW, Youn JI. Cross-sectional study on the correlation of serum uric acid with disease severity in Korean patients with psoriasis. Clin Exp Dermatol 2011;36:473-8.

15. Billiet L, Doaty S, Katz JD, Velasquez MT. Review of hyperuricemia as new marker for metabolic syndrome. ISRN Rheumatol 2014;2014:852954.

16. Gonzalez-Gay MA, Gonzalez-Juanatey C, Vazquez-Rodriguez TR, Dierssen T, Llorca J. Role of asymptomatic hyperuricemia and serum uric acid levels in the pathogenesis of subclinical atherosclerosis in psoriatic arthritis: comment on the article by Chen et al. Arthritis Rheum 2009;61:856-7.
17. Gonzalez-Gay MA, Gonzalez-Juanatey C, Vazquez-Rodriguez TR, Gomez-Acebo I, Miranda-Filloy JA, Paz-Carreira J, et al. Asymptomatic hyperuricemia and serum uric acid concentration correlate with subclinical atherosclerosis in psoriatic arthritis patients without clinically evident cardiovascular disease. Semin Arthritis Rheum 2009;39:157-62.

18. Gladman DD, Shuckett R, Russell ML, Thorne JC, Schachter RK Psoriatic arthritis (PSA) - an analysis of 220 patients. Q J Med 1987;62:127-41.

19. Taylor W, Gladman D, Helliwell P, Marchesoni A, Mease P, Mielants H; CASPAR Study Group. Classification criteria for psoriatic arthritis: development of new criteria from a large international study. Arthritis Rheum 2006;54:2665-73.

20. Gisondi P. Hyperuricemia in patients with chronic plaque psoriasis. Drug Dev Res 2014;75 Suppl 1:S70-2.

21. Azfar RS, Gelfand JM. Psoriasis and metabolic disease: epidemiology and pathophysiology. Curr Opin Rheumatol 2008;20:416-22.

22. Nejatinamini S, Ataie-Jafari A, Qorbani M, Nikoohemat S, Kelishadi R, Asayesh H, et al. Association between serum uric acid level and metabolic syndrome components. J Diabetes Metab Disord 2015;14:70.

23. Lyngdoh T, Marques-Vidal P, Paccaud F, Preisig M, Waeber G, Bochud M, et al. Elevated serum uric acid is associated with high circulating inflammatory cytokines in the population-based Colaus study. PLoS One 2011;6:e19901

24. Ibrahim SE, Helmi A, Yousef TM, Hassan MS, Farouk N. Association of asymptomatic hyperuricemia and endothelial dysfunction in psoriatic arthritis. Egypt Rheumatologist 2012; 34:83-9.

25. Kim S, Chang Y, Yun KE, Jung HS, Lee SJ, Shin H, et al. Development of nephrolithiasis in asymptomatic hyperuricemia: a cohort study. Am J Kidney Dis 2017;70:173-81.

26. Scarpa R, Cosentini E, Manguso F, Oriente A, Peluso R, Atteno M, et al. Clinical and genetic aspects of psoriatic arthritis "sine psoriasis". J Rheumatol 2003;30:2638-40.

27. Filiopoulos V, Hadjiyannakos D, Vlassopoulos D. New insights into uric acid effects on the progression and prognosis of chronic kidney disease. Ren Fail 2012;34:510-20.

28. Ogdie A, Yu Y, Haynes K, Love TJ, Maliha S, Jiang Y, et al. Risk of major cardiovascular events in patients with psoriatic arthritis, psoriasis and rheumatoid arthritis: a population-based cohort study. Ann Rheum Dis 2015;74:326-32.

29. Ernste FC, Sanchez-Menendez M, Wilton KM, Crowson CS, Matteson EL, Maradit Kremers H. Cardiovascular risk profile at the onset of psoriatic arthritis: a population-based cohort study. Arthritis Care Res 2015;67:1015-21.

30. Eder L, Wu Y, Chandran V, Cook R, Gladman DD. Incidence and predictors for cardiovascular events in patients with psoriatic arthritis. Ann Rheum Dis 2016;75:1680-6.

31. Li L, Yang C, Zhao Y, Zeng X, Liu F, Fu P. Is hyperuricemia an independent risk factor for new-onset chronic kidney disease?: A systematic review and meta-analysis based on observational cohort studies. BMC Nephrol 2014;15:122.

32. Wei L, Mackenzie IS, Chen Y, Struthers AD, MacDonald TM. Impact of allopurinol use on urate concentration and cardiovascular outcome. Br J Clin Pharmacol 2011;71:600-7. 